\title{
Do radiographic features of acute infection influence management of lower respiratory tract infections in the community?
}

\author{
J.C.G. Simpson*, P. Hulse+, P.M. Taylor+, M. Woodhead++
}

Do radiographic features of acute infection influence management of lower respiratory tract infections in the community? J.C.G. Simpson, P. Hulse, P.M. Taylor, M. Woodhead. CERS Journals Ltd 1998.

ABSTRACT: Community-acquired lower respiratory tract infection (LRTI) is a common clinical problem, and a chest radiograph is the most common investigation. This study investigated why general practitioners (GPs) request radiographs and whether radiographic features of $L R T I$ affect management.

The 12 month study included GP-requested chest radiographs performed at the Manchester Royal Infirmary. The GPs of patients with radiographic evidence of LRTI were sent a questionnaire.

Radiographs $(n=2,538)$ were performed in patients aged $>15$ yrs. One hundred and eleven $(4.4 \%)$ chest radiographs showed evidence of LRTI, and $97(87 \%)$ were included in the analysis. Fifty-six $(62 \%)$ had their radiograph requested at the second or third visit. Forty-five $(\mathbf{5 8 \%})$ radiographs were performed to confirm infection. In $47(48 \%)$ of cases the GP felt the radiograph had affected treatment. An effect on treatment was significantly more common in patients not receiving antibiotics prior to the radiograph. Twenty-nine (32\%) of GPs felt a normal radiograph would have affected management, most often with respect to antibiotic prescriptions (17 (58\%)). Where the radiologist's report suggested a repeat radiograph or hospital referral, this was significantly more likely to have occurred.

The commonest reason for a radiograph in patients with suspected lower respiratory tract infection is to confirm the infection. In the opinion of the general practitioner the results of the radiograph do affect patient treatment. The wording of the radiologists report is associated with patient management.

Eur Respir J 1998; 12: 1384-1387.
*North West Lung Centre, Wythenshawe Hospital, Manchester, UK. Depts of +Radiology and +Respiratory Medicine, Manchester Royal Infirmary, Manchester, UK.

Correspondence: M. Woodhead

Dept of Respiratory Medicine

Manchester Royal Infirmary

Oxford Road

Manchester

M13 9WL

UK

Fax: 441612764989

Keywords: Chest radiograph

general practice

respiratory infection

Received: March 111998

Accepted after revision August 11998
Community-acquired lower respiratory tract infection (LRTI) is a significant cause of both morbidity (approximately 1,700,000 cases annually in the UK between the age of 16 and $79 \mathrm{yrs}$ ) [1] and mortality. In addition, LRTIs are recognized to be "a substantial financial burden" to the health service as a result of investigation and treatment [2]. The term LRTI includes a wide range of diseases which have different underlying pathologies and aetiologies, e.g. acute bronchitis and pneumonia. Most episodes are managed in the community by general practitioners (GPs) without investigation or referral to a hospital. In a recent European survey [3], if investigations were performed (29\% of cases), chest radiography was the most common method (22\% of cases). The chest radiograph may be performed for a number of reasons, including confirmation of infection, assessment of response to treatment, looking for a complication of the infection and looking for underlying pathology such as a neoplasm. Pneumonia in hospitalized patients is often a radiological diagnosis, and conversely, in the community is often a clinical one. Previous investigators have identified factors predictive of radiographic pneumonia, such as focal chest signs $[4,5]$ and patients requiring a home visit [6]. The impact of a chest radiograph revealing radiographic features of pneumonia on the subsequent management by the GP is not known.

The aims of this study were to identify the proportion of chest radiographs performed by GPs to seek features of infection, and in those that show features of infection, to identify the timing and reason for performing the chest radiograph and the GP's opinion on whether the result influenced management.

\section{Methods}

Between April 1995 and March 1996 the reports of all chest radiographs performed at Manchester Royal Infirmary at the request of a GP were identified. From the radiograph report, the patient's age and GP were identified. Cases for study were selected if the patients were aged $>16$ yrs.

To identify the proportion of chest radiographs performed by GPs to seek features of infection, 100 consecutive radiographs requested by GPs were identified. A questionnaire was sent to each GP to assess the reasons for performing the radiograph.

To study the impact of radiographic evidence of acute infection, all chest radiographs (in the absence of other significant underlying pathology such as neoplasm) reported as showing evidence of "acute infection", "pneumonia" or "consolidation" were selected. A questionnaire requesting details regarding the indications for and the timing of the chest radiograph, was sent to the GP (fig. 1). Nonresponders were mailed a second time. 


\section{STUDY NO}

\section{PLEASE TICK THE APPROPRIATE BOXES}

1) At which visit was the CXR performed?

$$
\begin{aligned}
& \text { 1st } \\
& \text { 2nd } \\
& \text { 3rd or more }
\end{aligned}
$$

2) Was the patient thought to have a respiratory tract infection?

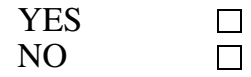

3) Was the patient thought to have pneumonia ?
YES
NO

4) Was the CXR performed to establish:

YES NO

The diagnosis of infection

Reason for lack of response to treatment

Possible underlying tumour

Other $\quad \square \quad$ please state

5) Did the findings on the CXR alter patient management ?



6) Was the patient receiving antibiotics prior to the CXR result ?

(a) YES $\quad \square \quad$ Length of course (days)

Which antibiotic

OR

Any changes following x-ray?

(b) NO $\quad \square \quad$ In the light of the report was one prescribed ?

YES $\square \quad$ Which?

7) Was a repeat CXR requested ?

YES

If yes how long after the first

$<4$ weeks

4-8 weeks

$>8$ weeks

NO

8) Did the CXR precipitate hospital referral ?

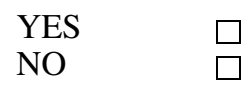

9) Would you have altered your treatment if the CXR had been normal ?

YES $\square \quad$ How ?

NO

Fig. 1. - Questionnaire sent to general practitioners, when chest radiography (CXR) revealed evidence of acute infection. 
Information was stored using dBase 3 (V.1.1; AshtonTate, Torrence, CA, USA) and statistical analysis was carried out by the Chi-squared test. The study was performed with the approval of the local research ethics committee and local medical advisory group.

\section{Results}

In total, 2,538 chest radiographs, requested by 58 different GPs, were performed during the 12-month period. In the sample of 100 consecutive chest radiographs, 47 $(51 \%)$ of the 92 responses indicated that chest radiograph had been performed to seek features of infection.

Features of infection, as defined in the Methods, were seen in $111(4.4 \%)$ of the 2,538 radiographs. Questionnaires were returned in 102 cases $(92 \%$ of the target population). Of these, 97 (87.4\%) were evaluable; for the others, either the form was returned blank or the GP stated that no radiograph report had been received.

The mean \pm SD age of the population (the 97 evaluable cases) was $58.9 \pm 20.3$ yrs (range 20-93 yrs). For the 91 cases where the GP provided information on the timing of the chest radiograph, $56(62 \%)$ were performed at the second or third visit to the GP for the illness in question. There was a nonsignificant trend for older patients to have a chest radiograph performed earlier (table 1). In 77 (79\%) of cases a LRTI was diagnosed by the GP before radiography. The reasons given for performing the chest radiograph in this group (with a clinically diagnosed LRTI) were to confirm infection in $45(58 \%)$; to assess treatment response in $33(43 \%)$; and to look for an underlying neoplasm in $30(39 \%)$. Assessment of treatment response was a more common indication at the third than at the first visit $(\mathrm{p}<0.001)$.

In $47(48 \%)$ of cases, in the opinion of the GP, the result of the chest radiograph had influenced management. If the GP reported that the radiograph did not alter management, patients were more likely to have been prescribed antibiotics before radiography (43 (86\%) versus $26(55 \%)$, p $<0.001$ ). In 29 (32\%) (of the 92 cases where question 9 was completed fully; see fig. 1) the GP reported that a normal result would have influenced management. Most commonly, this would have affected antibiotic prescriptions in 17 cases $(59 \%)$. Of the 32 repeat chest radiographs, 16 $(50 \%)$ were performed within 4 weeks of the first radiograph.

The most commonly prescribed antibiotic group was a penicillin/aminopenicillin in 41 cases $(59 \%)$. Fifty-six $(81 \%)$ of all antibiotic courses were for $<7$ days. Before the radiograph result $27(28 \%)$ patients were not receiving an antibiotic, despite $10(37 \%)$ of these being reported to have an LRTI by the GP. Excluding the two patients admitted directly to hospital as a result of the chest radiograph, eight patients at no point received an antibiotic from their GP, despite the radiograph report, five of whom were subsequently referred to the outpatient clinic.

Table 1. - Timing of chest radiograph performance related to visit and age

\begin{tabular}{llll}
\hline & Visit 1 & Visit 2 & Visit 3 \\
\hline \% Performed & 38 & 40 & 22 \\
Mean age yrs & 59.5 & 57.9 & 55.9 \\
\hline
\end{tabular}

Table 2. - Effect of the radiologist's report on hospital referral

\begin{tabular}{lccc}
\hline & & Hospital referrals recommended \\
& & Yes & No \\
\hline Referral to hospital & Yes & $11^{* * *}$ & 17 \\
& No & 0 & 69 \\
Total & & 11 & 86 \\
\hline$* * *: \mathrm{p}<0.001$. & &
\end{tabular}

The terms "infection", "consolidation" and "pneumonia" appeared in the radiologist's report in 67 (68\%), 63 (64\%) and $14(15 \%)$ cases, respectively. A single report may have contained any combination of one or more of these terms. The relationship between the wording of the report and the GP's response with respect to hospital referral and repeat radiography (excluding those admitted or referred to hospital) is shown in tables 2 and 3 . Both were more likely to have occurred if recommended by the radiologist's report: referral to hospital 100 versus $20 \%$ ( $p<0.001)$ and repeat radiography 78 versus $29 \%(\mathrm{p}<0.001)$.

\section{Discussion}

The response rates from both the target population and the sample drawn from the last month (92\%) were high, suggesting that statistically valid samples were collected. The simplicity and brevity of the questionnaire, in conjunction with the study appearing relevant to daily practice, may explain the high response rate. The accuracy of the data collected by the questionnaire is dependent on the accuracy of the GP's answers. The questionnaire was not formally examined for its reproducibility.

The results suggest that about $50 \%$ of requests for chest radiography were initiated to seek features of infection. Extrapolating this to the total number of chest radiographs performed over the year, approximately 1,300 were performed to look for infection. The proportion of these with radiographic features of acute infection was $8.5 \%$ (111 out of 1,300), a finding similar to previous studies [1]. This may be viewed as supporting the hypothesis that "chest radiography for GPs is a low yield investigation" [7]. However, this apparent low yield does not reflect the potential benefit of a "negative result" for the GP, as it may provide evidence against underlying lung disease or a complication resulting from infection. A negative result may also obviate the need for a relatively expensive hospital referral. The cost of the 2,538 chest radiographs over the 12 -month period was approximately $£ 75,000$ (assuming a cost of $£ 30$ per film).

In $47(48 \%)$ of the patients with radiographic features of acute infection the GP reported that the chest radiograph result had influenced patient management and that

Table 3. - Effect of the radiologist's report on repeat chest radiography

\begin{tabular}{lccc}
\hline & \multicolumn{3}{c}{ Repeat radiograph recommended } \\
& & Yes & No \\
\hline Repeat radiograph & Yes & $21^{* * *}$ & 11 \\
performed & No & 6 & 27 \\
Total & & 27 & 38 \\
\hline
\end{tabular}

$* * *: \mathrm{p}<0.001$. 
in one-third of cases, a normal radiograph would have affected treatment. It was beyond the scope of this study to assess objectively the appropriateness or otherwise of the GPs' responses to the radiographic result. Previous studies have suggested an influence of the radiographic result (revealing features of acute infection) on both antibiotic prescription and the course of illness [8]. The current study found that a chest radiograph showing evidence of infection was less likely to influence the management when patients were already receiving antibiotics and that a normal radiograph would have been likely to affect antibiotic treatment. It is difficult to comment on the eight individuals with a chest radiograph revealing features of acute infection who were not prescribed antibiotic treatment, since the time of arrival of the report in relation to the evolution of the clinical illness is not known. Chest radiographic shadowing in pneumonia is known to resolve more slowly than clinical recovery, so in a clinically recovered patient persisting radiographic consolidation may require no further action. As would be expected, assessment of the treatment response was found to be a significantly more common reason for performing radiography at the third than at the first visit. In the instances where a repeat radiograph was performed, $16(50 \%)$ were performed $<4$ weeks following the first. The optimum timing of repeat chest radiography is not known. Studies of patients hospitalized for community-acquired pneumonia indicate that an interval of 6 weeks is appropriate to allow more complete radiographic resolution [9]. However, the faster resolution of such shadowing in patients remaining in the community may indicate that 4 weeks is more appropriate [6].

All patients in whom hospital referral was recommended by the radiologist were referred and a repeat film was performed more frequently where a radiologist had suggested this. The study design does not allow the conclusion to be drawn that the chest radiography report was the reason for repeat radiography or hospital referral. Nor did it allow an examination of whether any of the individual terms, "infection", "consolidation" and "pneumonia" specifically affected management. However, the results suggest that the wording of the chest radiography report may play a significant role in the management of patients. Radiologists should be conscious of the power of their report in influencing the management of patients. Not all reports contain recommendations regarding repeat films and referral to hospital. This may reflect the usual reporting habits of individual radiologists or specific clinical or radiologi- cal indications. The GPs are unaware of which of these is the case when deciding on a management plan.

In conclusion, the most common reason for performing a chest radiograph in patients with lower respiratory tract infection was to look for features of infection. The results of chest radiographs requested by general practitioners, in patients with radiographic evidence of infection, have an impact on the management of the patients. In addition, in this group of patients, the wording of the radiograph report was significantly related to management. The study highlights a number of issues in relation to the use of chest radiographs in lower respiratory tract infection in the community which might form a suitable basis for future studies.

\section{References}

1. Macfarlane JT, Colville A, Guion A, Macfarlane RM, Rose DH. Prospective study of aetiology and outcome of adult lower-respiratory-tract infections in the community. Lancet 1993; 341: 511-514.

2. Guest JF, Morris A. Community-acquired lower respiratory tract infections: the annual cost to the National Health Service. Br J Med Econ 1996; 10: 263-273.

3. Woodhead M, Gialdroni Grassi G, Huchon GJ, Leophonte $\mathrm{P}$, Manresa F, Schaberg T. Use of investigations in lower respiratory tract infection in the community: a European survey. Eur Respir J 1996; 9: 1596-1600.

4. Woodhead MA, Macfarlane JT, McCracken JS, Rose DH, Finch RG. Prospective study of the aetiology and outcome of pneumonia in the community. Lancet 1987; 1 : 671-674.

5. Macfarlane JT, Colville A, Guion A, Macfarlane R, Rose DH. Adult lower respiratory tract infections (LRTI): which investigations are useful to GPs? Thorax 1993; 48: A462.

6. Woodhead MA. Studies on pneumonia in the community and in hospital in Nottingham. MD Thesis, Nottingham, 1988.

7. Guyer PB, Chalmers AG. Chest radiography for general practitioners-a low yield investigation. J R Coll Gen Pract 1983; 33: 477-479.

8. Bushyhead JB, Wood RW, Tompkins RK, Wolcott BW, Diehr P. The effect of chest radiographs on the management and clinical course of patients with acute cough. Med Care 1983; 21: 661-673.

9. Macfarlane JT, Miller AC, Smith WHR, Morris AH, Rose DH. Comparative radiographic features of community acquired legionnaires disease, pneumococcal pneumonia, mycoplasma pneumonia and psittacosis. Thorax 1984; 39: 28-33. 